\title{
Analysis of the Hamstring Muscle Activation During two Injury Prevention Exercises
}

\author{
by \\ Alireza Monajati ${ }^{1}$,Eneko Larumbe-Zabalaㄹ, Mark Goss-Sampson ${ }^{1}$, \\ Fernando Naclerio $^{1}$
}

The aim of this study was to perform an electromyographic and kinetic comparison of two commonly used hamstring eccentric strengthening exercises: Nordic Curl and Ball Leg Curl. After determining the maximum isometric voluntary contraction of the knee flexors, ten female athletes performed 3 repetitions of both the Nordic Curl and Ball Leg Curl, while knee angular displacement and electromyografic activity of the biceps femoris and semitendinosus were monitored. No significant differences were found between biceps femoris and semitendinosus activation in both the Nordic Curl and Ball Leg Curl. However, comparisons between exercises revealed higher activation of both the biceps femoris $(74.8 \pm 20$ vs $50.3 \pm 25.7 \%, p=0.03 d=0.53)$ and semitendinosus $(78.3 \pm 27.5$ vs $44.3 \pm 26.6 \%, p=0.012, d=0.63)$ at the closest knee angles in the Nordic Curl vs Ball Leg Curl, respectively. Hamstring muscles activation during the Nordic Curl increased, remained high $(>70 \%)$ between 60 to $40^{\circ}$ of the knee angle and then decreased to $27 \%$ of the maximal isometric voluntary contraction at the end of movement. Overall, the biceps femoris and semitendinosus showed similar patterns of activation. In conclusion, even though the hamstring muscle activation at open knee positions was similar between exercises, the Nordic Curl elicited a higher hamstring activity compared to the Ball Leg Curl.

Key words: semitendinosus, biceps femoris, Nordic Curl, Ball leg curl, female soccer players.

\section{Introduction}

The hamstrings, comprising biceps femoris (BF), semitendinosus (ST) and semimembranosus $(\mathrm{SM})$, compose a bi-articular muscle group crossing the hip and knee joint that acts synergistically in extending the hip and flexing the knee during sprints related activities (Opar et al., 2012). Hamstrings are highly activated in sports involving deceleration, acceleration and jumping (Arnason et al., 2008) and represent one of the most frequently injured muscle groups in soccer (Monajati et al., 2016; Woods et al., 2004). Despite the complex aetiology, the occurrence of hamstring strain injury (HIS) is associated with rapid actions involving hip flexion and knee extension, when the muscles are subject to high forces in combination

with rapid muscle lengthening (Opar et al., 2012). In sprinting, HIS occurs when hamstrings are actively lengthened and contract to decelerate the thigh and the lower leg to an angle of approximately $30^{\circ}$ before extending the knee during the last half of the swing phase (Ditroilo et al., 2013; Heiderscheit et al., 2005). It is widely suggested that the repetition of fast eccentric muscle actions toward open knee angles results into accumulated microscopic muscle damage that may develop into an injury (Timmins et al., 2015)

Over the last decade, a large number of studies have investigated the effectiveness of injury prevention exercises in eliciting specific physiological adaptations aimed to attenuate

\footnotetext{
1 - Department of Life and Sports Science, University of Greenwich. Medway Campus, Kent (UK).

2 - Clinical Research Institute, Texas Tech University Health Sciences Center, Lubbock, TX (USA).
} 
sarcomere damage during repeated active lengthening actions (Brockett et al., 2001) along with an increase of hamstring strength at different knee angular positions (Opar et al., 2012). In addition to free weight and machine resistance exercises like dead lift (Heiderscheit et al., 2010; Timmins et al., 2015), trunk hyperextension or leg curl (Holcomb et al., 2007; Pollard et al., 2006), hamstring eccentric exercises (HEEs) using no external load such as Nordic Curl (NC) (Clark et al., 2005; Lim et al., 2009; Mjolsnes et al., 2004) and Ball Leg Curl (BLC) (Holcomb et al., 2007; Ortiz et al., 2010) have been proposed to be effective for increasing eccentric hamstring strength. Advantages of weight bearing exercises are as follows: 1) no additional equipment or facilities are required thus making the program easy to follow, 2) they simulate the activity of daily living and 3) simulate the same tension on muscles that may occur during a sport activity. These advantages have prompted coaches to use weight-bearing exercises as a part of injury prevention protocols (Farrokhi et al., 2008). Conversely, the use of weight bearing exercises would not allow for individualised control of the overload, nor the application of a more intense stimulus that could be obtained through a progressive protocol using external resistance such as dumbbells or weight vests.

Despite the aforementioned proposed effectiveness of NC and BLC for preventing HSI, there is still a paucity of research that compares the differential level of activation of the individual hamstring muscles throughout the open knee angles during these injury prevention exercises.

Ditroilo et al. (2013) reported a higher level of BF activation during NC compared to a traditional maximal eccentric exercise performed on an isokinetic machine. However, in this study no other hamstring muscles were analysed. Iga et al. (2012) reported significant eccentric peak torque improvements and an increased capability to resist lengthening actions at more extended joint positions of the hamstrings of both limbs during NC after a 4-week progressive exercise program involving only NC. More recently, Marshall et al. (2015) observed a statistically significant decrease in BF activation, but not of ST, during a 6-set of 5 repetitions NC-only exercise bout in 10 soccer players.

To the best of the authors' knowledge, no study so far has analysed and compared the patterns of hamstring activation over the knee open angles, where the majority of HSIs occur, in two different exercises. Such an investigation would allow researchers, clinicians and coaches to quantify and monitor the training-related adaptations based on kinematic and electromyographic analysis. Therefore, the aim of the present study was twofold: (a) to analyse the pattern of eccentric hamstring activation of two commonly used hamstring strengthening exercises, NC and BLC, by measuring the activity of the BF and ST with respect to knee angles, (b) to determine differences in the level of BF and ST muscle activation between NC and BLC exercises. The achievement of the aforementioned objectives will allow coaches to determine whether the two analysed exercises are appropriate for strengthening the hamstrings at more open length and consequently protecting athletes from hamstring injuries.

\section{Methods}

\section{Procedures}

This study utilised a single-group repeated measures design, where 2 within-participant conditions, i.e. NC and BLC, were examined. Once considered eligible for the study, participants were required to attend the laboratory on two different occasions. On the first visit participants were assessed for body mass and height. In addition they were familiarised with both NC and BLC exercises. The second visit required participants' determination of the maximum voluntary isometric contraction (MVIC) before performing the NC and BLC exercise. The muscle activity of the BF and ST was monitored through the root mean square (RMS) surface electromyography signal amplitude (EMGs). To maintain a suitable balance between different possible order of treatments and minimise any confounding effects, the order of exercises was randomised in a controlled manner. Thus, half of the participants started with the NC and half with the BLC. The study was carried out in accordance with the guidelines contained in the Declaration of Helsinki and was approved by the University of Greenwich Research Ethics Committee.

\section{Participants}

Ten female soccer players from the English Women's Super League, second division (mean \pm 
SD age $22 \pm 4.7 \mathrm{yrs}$, body mass $56 \pm 4.8 \mathrm{~kg}$ and body height $163 \pm 5.4 \mathrm{~cm}$ ) participated in this study. All participants were engaged in regular soccer training ( 3 sessions per week) for a minimum of 6 years and used resistance exercises as an essential component of their conditioning preparation during the last 12 months before the beginning of the study. Participants were excluded if they had: 1) hamstring injuries 6 months prior to the study; 2) history of knee injury; or 3) participated in any hamstring injury prevention program during the last 12 months prior to the study. Before participating in this study, all players read and signed an informed consent form. They were also asked to refrain from caffeine ingestion and any unaccustomed or hard exercise during the $72 \mathrm{~h}$ before the assessment sessions.

\section{Measures}

\section{Exercises description}

Three trials of the NC and BLC were completed in randomised order. On the first visit participants were familiarised and shown the correct technique for each exercise. During the next visit they performed both exercises and received individual feedback. The remaining visit comprised the testing session that consisted of a 10 min warm up involving dynamic stretching, jogging, running and jumping exercises. Participants had $30 \mathrm{~s}$ rest between trials and $2 \mathrm{~min}$ rest between exercises to allow full recovery.

Nordic Curl

Participants began by kneeling on the floor with the upper body vertical and straight with the knee flexed to $90^{\circ}$ and hip fully extended. A partner applied pressure on the heels in order to make sure that the feet kept contact with the floor throughout the movement. The participants began moving their upper body forward while keeping their hip extended (avoiding hyperextension) and slowly lowered their upper body and extended their knee trying to resist the fall by contracting their hamstring muscles. Arms were kept flexed with hands by the shoulders as long as possible and they would be pushed forward only if necessary to buffer the fall avoiding a violent landing of the body onto the ground at the final stages of the movement (Figure 1A).

Ball leg curl

Participants began by lying supine on the floor with their heels on the ball, knee extended and hands on the floor by their sides, palm facing down. They were asked to simultaneously flex their knee while rolling the ball toward themselves and lifting their pelvis from the ground to form a plank and maintain this position for about $1 \mathrm{~s}$ before slowly returning to the starting position by simultaneously extending the knee and lowering the pelvis (Figure 1B).

\section{sEMG and Kinematic data collection}

The dominant (preferred kicking) limb was selected for data collection. Prior to electrode placement, the skin was shaved abraded and cleaned with isopropyl alcohol. Parallel-bar EMG Sensors (DE-2.1, DELSYS, USA) were then placed over the BF and ST in accordance to SENIAM guidelines (Hermens et al. 2000). EMG signals were amplified (1 k gain) via a Delsys Bagnoli system (Delsys Inc. Boston, MA, USA) with a bandwidth of $20-450 \mathrm{~Hz}$. The common mode rejection rate and input impedance were $-92 \mathrm{~dB}$ and $>10^{15} \Omega$, respectively. Data was collected at $1000 \mathrm{~Hz}$ synchronously with the kinematic data.

Lower extremity planar kinematics was monitored using a 10-camera retroreflective system at $200 \mathrm{~Hz}$ (Oqus 3, Qualisys Gothenburg, Sweden). Four retroreflective soft markers (19 mm) were placed over the lateral malleolus, lateral knee joint, greater trochanter and acromion process of the dominant limb. Following tracking, kinematic and sEMG data were exported for analysis to Visual 3D (C-Motion Inc. USA).

\section{Data processing}

Sagittal plane knee angles were derived in Visual3D and all data processed in this trial was based on analysis within $20^{\circ}$ movement epochs. For the purpose of this study, the exercises were analysed during the eccentric phase and over the knee open angles $\left(>60^{\circ}\right)$. As a consequence each exercise was divided into 3 phases (phase 1, 60-40 ; phase $2,40-20^{\circ}$; phase $3,20-0^{\circ}$ ) where 0 was defined as a fully extended knee joint. For each phase the root mean square (RMS) of the EMG amplitude data was calculated and then low pass filtered with the cut-off frequency of $6 \mathrm{~Hz}$. The start of each phase for NC and BLC exercises was confirmed from the knee angle (Figure 1). Briefly, the RMS is the square root of the arithmetic mean of the square values of the EMG signal and was measured according to Equation 1.

$$
\chi_{r m s}=\sqrt{\sum x_{n}^{2}}
$$


where $\mathrm{X}_{\text {rms }}$ is the computed EMGrms value, $\mathrm{T} 1$ and $\mathrm{T} 2$ are the start and finish times of each contraction burst. Data were collected from $60^{\circ}$ until the participants completed the eccentric phase for both the NC and BLC.

\section{sEMG normalization procedure}

In order to compare values of different muscle activation patterns, sEMG data were normalised as a percentage of the EMG signal recorded during a dominant leg maximum isometric voluntary contraction of the knee flexors (MVIC). The MVIC test was performed with participants in the prone position with knees flexed to $30^{\circ}$ (anatomical angle). The MVIC was held for $5 \mathrm{~s}$ and the peak $3 \mathrm{~s}$ of the EMG signal were used for normalization purposes. The muscle activity of the BF and ST was recorded and considered the reference value for normalizing EMGs measured during the NC and LBC tests.

\section{Statistical analysis}

A descriptive analysis was performed and subsequently the Kolmogorov-Smirnov and
Shapiro-Wilk test were applied to assess normality. Two independent $2 \times 3$ mixed analysis of variance (ANOVA) models, one per exercise (NC and BLC), were performed in order to determine differences in muscle activation between muscles (BF vs ST) over the three phases. Furthermore, two independent $2 \times 3$ mixed ANOVA models, one per muscle, were performed to determine differences in muscle activation between exercises and over the three phases.

Generalised eta squared $\left(\eta_{G}^{2}\right)$ and Cohen's $d$ values were reported to provide an estimate of standardised effect size (small $\mathrm{d}=0.2, \eta_{G}^{2}=0.01$; moderate $\mathrm{d}=0.5, \eta_{G}^{2}=0.06$; and large $\mathrm{d}=0.8, \eta_{G}^{2}=$ 0.14 ). The level of significance was set at $p<0.05$ for all tests.

\section{Results}

No main effects were observed between the activation of the BF and ST across the three analysed phases for both exercises, NC $(\mathrm{F}(1,18)=$ $0.046, p=0.833)$ and $\operatorname{BLC}(\mathrm{F}(1,18)=0.387, p=0.542)$.

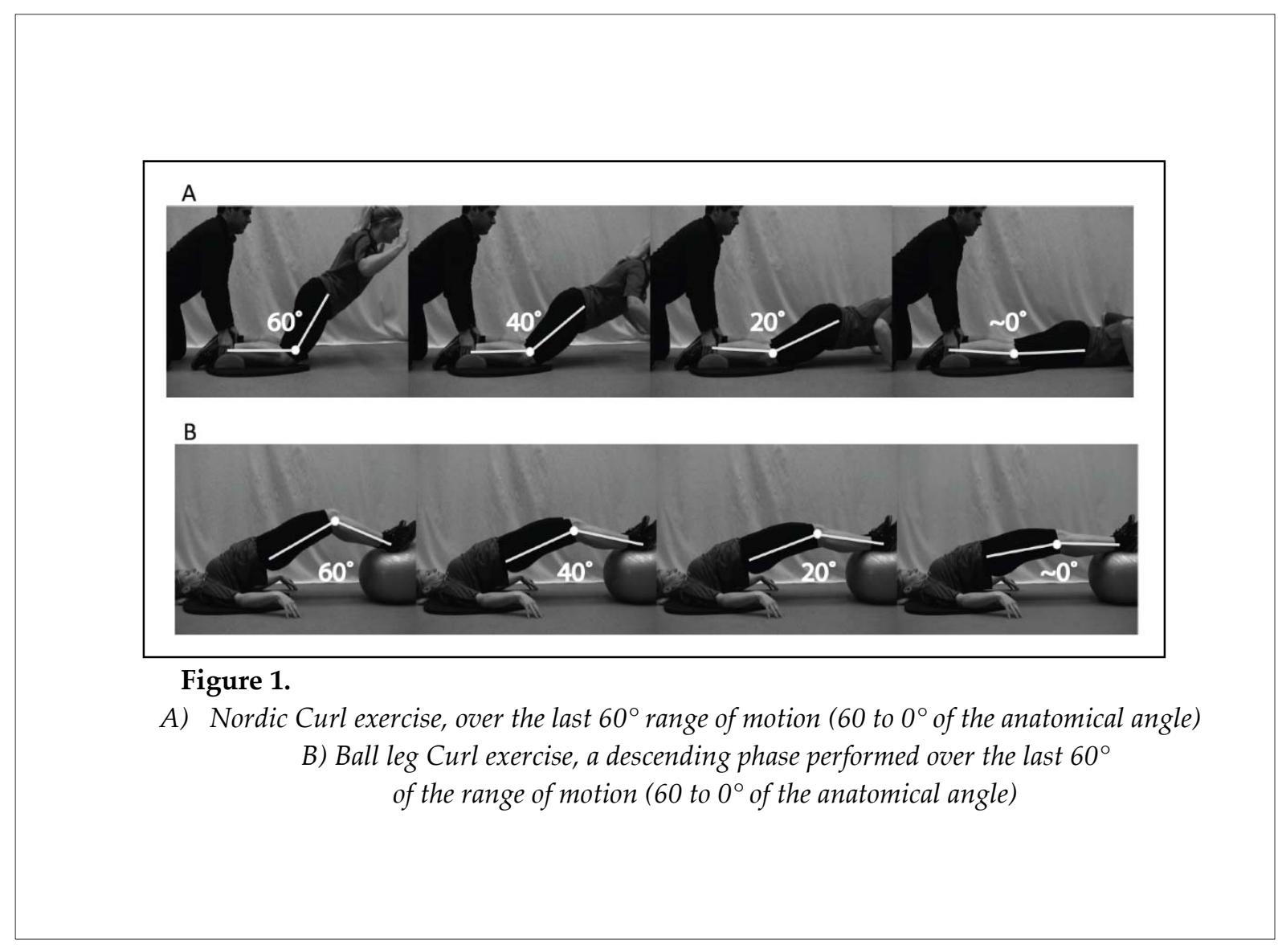




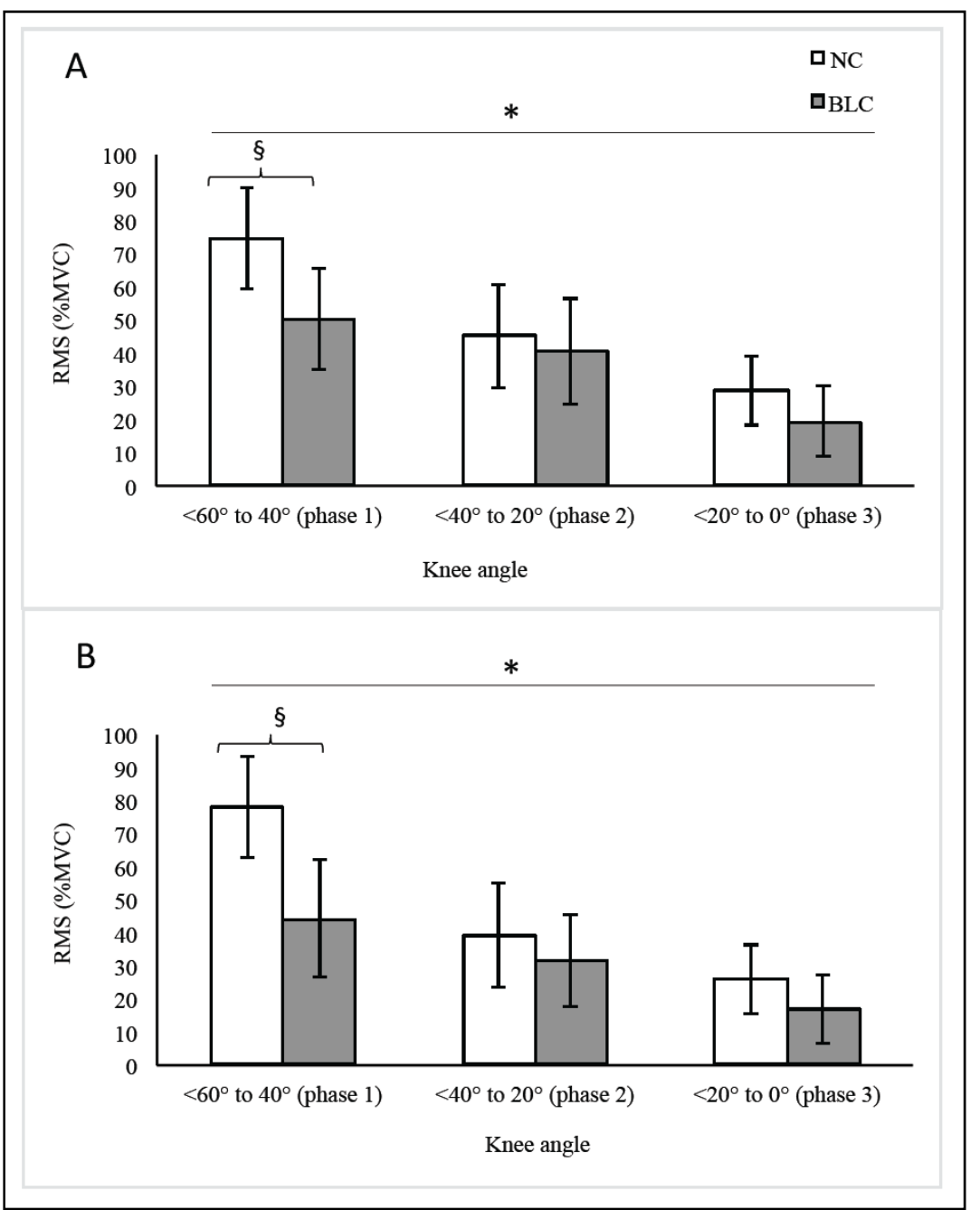

Figure 2.

A) Biceps Femoris activation during Nordic Curl (NC) and Ball Leg Curl (BLC). (Mean $\pm 95 \%$ confidence intervals). ${ }^{*} p<0.001$ between phases 1 vs $2 ; 1$ vs 3 and 2 vs 3 for NC as well as 1 and 2 vs 3 in for BLC. $\S p=0.03$ between NC and BLC at phase 1 .

B) Semitendinosus activation during Nordic Curl (NC) and Ball Leg Curl (BLC).

(Mean $\pm 95 \%$ confidence intervals). ${ }^{*} p<0.001$ between phases 1 vs $2 ; 1$ vs 3 and 2 vs 3 for NC and BLC. $\S p=0.012$ between NC and BLC at phase 1

\section{Biceps Femoris Activation}

No significant effect between exercises $\left(\mathrm{F}(1,18)=2.20, p=0.155, \eta_{G}^{2}=0.09\right)$ or interaction effects were determined for exercise and phases $\left(\mathrm{F}(1,18)=3.42, p=0.081, \eta_{G}^{2}=0.02\right)$. However, a significant main effect between phases $(\mathrm{F}(1,18)=$
87.08, $\left.p<0.001, \eta_{G}^{2}=0.36\right)$ was determined. Pairwise comparisons revealed significant differences $(p<0.001)$ and large effect sizes (phase 1 vs. $2, \mathrm{~d}=1.38$; phase 1 vs. $3, \mathrm{~d}=1.78$ and phase 2 vs. $3, d=0.86$ ) for the NC. A similar pattern was determined for the BLC, where the activation of the 
BF during both phase $1(p<0.001, \mathrm{~d}=1.19)$ and $2(p$ $<0.001, \mathrm{~d}=1.11)$ was significantly higher than in phase 3 , and a strong trend with a moderate effect size to produce a higher activation during the phase 1 compared to phase 2 was also determined $(p=0.058, d=0.45)$. Furthermore, the activation of the BF during phase 1 was significantly higher in the NC compared to the BLC $(74.8 \pm 20$ vs $50.3 \pm$ $25.7 \%, p=0.03, \mathrm{~d}=0.53$ ) (Figure $2 \mathrm{~A}$ ).

\section{Semitendinosus Activation}

Significant phase effects $(\mathrm{F}(1,18)=50.79, p<$ $\left.0.001, \eta_{G}^{2}=0.34\right)$ and interaction effects between phases and exercises $\left(\mathrm{F}(1,18)=4.91, p=0.040, \eta_{G}^{2}=\right.$ $0.05)$ were observed. However, no main effects between exercises were determined $(\mathrm{F}(1,11)=4.05$, $\left.p=0.060, \eta_{G}^{2}=0.14\right)$. Pairwise comparisons revealed significant differences and large to moderate effect sizes for both analysed exercises, i.e. $\mathrm{NC}(p<0.001$, phase 1 vs. $2, \mathrm{~d}=1.58$; phase 1 vs. $3, \mathrm{~d}=1.48$ and phase 2 vs. $3, \mathrm{~d}=0.86$ ) and BLC (phase 1 vs. $2 p=0.036, \mathrm{~d}=0.51$; phase 1 vs. $3, p=$ $0.003, d=0.78$ and phase 2 vs. $3, p<0.001, d=0.96$ ). Furthermore, the activation of the ST during phase 1 was significantly higher in the NC than in the BLC $(78.3 \pm 27.5$ vs $44.3 \pm 26.6 \%, p=0.012, d=0.63)$ (Figure 2B).

\section{Discussion}

The main finding of the present study showed that for uninjured female soccer players the pattern of ST and BF activation during both the NC and BLC was similar throughout the knee open angles over the eccentric displacement. However, when comparing the level of muscular activation elicited by each exercise, the following differences were identified: 1 ) at the closest knee angle position $\left(60-40^{\circ}\right)$ the activation of both the BF $(74.8 \pm 20 \mathrm{vs}$ $50.3 \pm 25.7 \%)$ and ST $(74.8 \pm 20$ vs $50.3 \pm 25.7 \%)$ was greater in the NC compared to the BLC; 2) during the NC, the activation of hamstring remained high from 60 to $40^{\circ}(\sim 77 \%$ of the MVIC) and then significantly decreased from $40^{\circ}$ to full extension (from $77 \%$ to $27 \%$ of the MVIC) and 3 ) the activation of hamstring was similar between the $\mathrm{NC}$ and BLC at the most extended angles $\left(<40^{\circ}\right)$.

Results from the present study provide an important insight into the understanding of the pattern of hamstring activation throughout the eccentric phase of the NC and BLC. The present investigation supports the finding of Zebis et al. (2013) who reported a very similar activation of the medial (ST) and lateral (BF) hamstrings during the NC and supine bridging exercises. The ST and $\mathrm{BF}$ have the ability to counteract the frontal plane applied force and help prevent an exaggerated knee varus and valgus mechanism during landing or changes of direction activities (Hubley-Kozey et al., 2006). Although the NC and BLC require a similar BF and ST activation, due to a shorter moment arm of the BF, the capacity of these muscles to generate torque is not equal (Lynn and Costigan, 2009). Therefore, in order to balance the force applied on the frontal plane, the BF must generate greater force compared to the ST. Due to this inherent imbalance, performing BF dominated exercises, such as hip extension and supine leg curl (Zebis et al., 2013), may help to achieve a balance between ST and BF torques in the frontal plane. Such enhancement in the balance between hamstrings torque on the frontal plane may help to prevent HSI, improve knee stabilization and consequently reduce the risk of other knee-related injuries, such as anterior cruciate ligament laceration (Stevenson et al., 2015).

It is widely accepted that hamstring weakness and muscle imbalances increase the risk of HSI in athletes. Thus, hamstring-strengthening exercises should be considered as an essential component of the injury prevention programmes (Orchard et al., 1997; Thelen et al., 2005). The relative load applied to the musculoskeletal system positively influences strength. Heavy loads (3-5 RM) are associated with greater strength gains compared to lighter loads (9-11 RM) (Campos et al., 2002). The relative load recommended for novice and advanced individuals to improve muscle strength is about $60-70 \%$ and $80-100 \%$ of 1 RM, respectively (Guex and Millet, 2013). Our results indicated that during the $\mathrm{NC}$, hamstring activity was significantly higher over the first phase $\left(60-40^{\circ}\right)$ of the range of motion and therefore, the NC would result in greater strength enhancement compared to the BLC. Even though hamstring activation of the two analysed exercises (NC and BLC) remained high from 60 to $40^{\circ}$ knee angles, and then progressively declined toward the end of the movement, the observed decline was higher for the NC. These findings are in line with those reported by Ditroilo et al. (2013) who observed a control of the downward movement during the first half of the range of motion and peak velocity of the downward movement 
occurred at $44^{\circ}$ of the knee angle. The above findings suggest that the NC exercise would be divided into the following two parts:

Part 1, from 60 to $40^{\circ}$ knee angle (phase 1), where the movement is controlled, hamstring muscles resist knee extension and decelerate the downward movement of the trunk. Thus hamstrings are highly activated along with an eccentric controlled muscle action that peaked at the middle of the range of motion $\left(60\right.$ to $\left.40^{\circ}\right)$.

Part 2, from the middle of the range of motion (knee angle $40^{\circ}$ ) until the end of the movement where the trunk approaches the ground (phases 2 and 3). As the trunk moves forward, the movement becomes progressively uncontrolled. The hamstring moment arm is shortening while the body mass moment arm is gradually lengthening $\left(41 \%\right.$ and $73 \%$ from $60^{\circ}$ to $45^{\circ}$ and $60^{\circ}$ to $30^{\circ}$, respectively). Due to this biomechanical disadvantage, it is expected that hamstring activation will increase to overcome the greater load as the trunk leans forward. However, it is important to highlight that our results show a decreased hamstring activation during the last $40^{\circ}$. Therefore, the hamstrings fail to attenuate the increased torque and the downward moment is accelerated.

During the NC, the hamstring acts at the hip and knee simultaneously to resist knee extension as well as hip flexion. One possible explanation for the decreased hamstring activity during the late phase of the $\mathrm{NC}$ may be due to the high biomechanical disadvantage observed during the last $40^{\circ}$ of the movement as hamstrings act mainly at the hip level to retain full hip extension and prevent uncontrolled falls. Furthermore, it is also possible that during the second part of the movement (phases 2 and 3), as the torque produced at the knee increases and overcomes the hamstring peak torque, the muscles cease resisting against the knee torque in order to avoid muscle strain and only act at the hip to prevent hip flexion. Therefore, the pattern of hamstring activation during the two aforementioned parts is distinctly different. During the first part the hamstring contracts to break knee extension, while during the second part the hamstring resists the hip flexion. Although speculative, it could be possible to hypothesize that as the capacity of the hamstring to apply force improves and its peak torque increases and shifts toward more flexed knee angles, the extension of the second part would progressively be reduced. Thus, before using the $\mathrm{NC}$, coaches should consider the use of methodological exercise progression starting with relatively low demanding exercises as LBC or assisted Nordic Curl with a band attached to the participant's back in order to facilitate control of the overload during the last part of the range of motion (Naclerio et al., 2015).

Results of the present study also indicate a similar level of muscle activation $(<45 \%$ of the MVIC) during the last $40^{\circ}$ knee angles between the NC and BLC. It is widely accepted that the majority of HSI occur during the late swing phase of the sprint where the knee is at the more extended angle position $\left(<40^{\circ}\right)$ (Guex and Millet, 2013; Heiderscheit et al., 2005). Thus, in order to prevent athletes from HSI, it is crucial to increase the overall hamstring strength, emphasising the capacity to apply force over the more extended knee angles. Nonetheless, the present results do not enable to evaluate the pattern of muscle activation when performing a typical injury prevention programme involving 3 to 5 exercises of 8 to 10 repetitions, or whether the level of muscle activation measured at the most extended angles by the two exercises is sufficient to reduce the incidence of HSI in athletes.

During the eccentric phase of both analysed exercises, NC and BLC, hamstring muscles actively lengthen while the hip is fully extended $\left(\sim 0^{\circ}\right)$ and the knees extend from $60^{\circ}$ until the full extension position $\left(\sim 0^{\circ}\right)$. However, during the late swing phase of a sprint cycle, the hip and knees are flexed to about $55-65^{\circ}$ and $30-40^{\circ}$, respectively. Due to a greater hamstring moment arm determined at the hip compared to the knee, the effect of changing the hip angle on BF and ST length is much greater than that at the knee angle (Visser et al., 1990). Therefore, during the late swing phase, where the hip is flexed, the hamstring muscles achieve a higher overall stretch compared to the exercises analysed in the present study (NC and BLC). In addition, during the NC and BLC, knees extend progressively along with an extended hip, therefore hamstring muscles contract within their nominal upright length.

\section{Conclusions}

The NC exercise elicited a higher level of hamstring activation compared to the BLC. The 
level of muscle activation during the NC (70-80\% of the MVIC) suggests that performing the NC exercise would enhance hamstring muscle strength. In addition, the level of BF and ST activation was similar throughout the range of motion, which indicates that using any of the analysed exercises may not result in muscle imbalances between the BF and ST.

During the NC and BLC, hamstring muscles activate within their resting length and therefore, it is not clear whether the analysed exercises would have the ability to simulate a similar pattern of muscle activation as occurred during hamstring strain related injuries, where muscles lengthen beyond their upright length.

\section{Limitations}

The reference values for the muscle activity elicited during the analysed exercise were presented in terms of the percentage of the MVIC measured with knees flexed to $30^{\circ}$ (open angle). Therefore it is not possible to evaluate whether the percentage of muscle activation produced by the tested exercises would be similar to that produced during the late swing phase of a sprint cycle, where the majority of hamstring injuries occur (Thelen et al., 2005).

Further investigations, using sprint as a reference exercise, would be needed in order to evaluate the relative degree of hamstring activation elicited by different proposed hamstring strengthening exercises.

\section{Acknowledgements}

The authors gratefully acknowledge the players of the Millwall Lionesses Ladies Football Club for their cooperation as participants of the current investigation.

The University of Greenwich provides funding to one of the author's Ph.D. project, however this does not affect this original research article and its content.

\section{References}

Arnason A, Andersen TE, Holme I, Engebretsen L, Bahr R. Prevention of hamstring strains in elite soccer: an intervention study. Scand J Med Sci Sports, 2008; 18(1): 40-48

Brockett CL, Morgan DL, Proske U. Human hamstring muscles adapt to eccentric exercise by changing optimum length. Med Sci. Sport Exerc, 2001; 33(5): 783-790

Campos GE, Luecke TJ, Wendeln HK, Toma K, Hagerman FC, Murray TF, Ragg KE, Ratamess NA, Kraemer WJ, Staron RS. Muscular adaptations in response to three different resistance-training regimens: specificity of repetition maximum training zones. Eur J Appl Physiol, 2002; 88(1-2): 50-60

Clark R, Bryant A, Culgan JP, Hartley B. The effects of eccentric hamstring strength training on dynamic jumping performance and isokinetic strength parameters: a pilot study on the implications for the prevention of hamstring injuries. Phys Ther Sport 2005; 6: 67-73

Ditroilo M, De Vito G, Delahunt E. Kinematic and electromyographic analysis of the Nordic Hamstring Exercise. J Electromyogr Kinesiol, 2013; 23(5): 1111-1118

Farrokhi S, Pollard CD, Souza RB, Chen YJ, Reischl S, Powers CM. Trunk position influences the kinematics, kinetics, and muscle activity of the lead lower extremity during the forward lunge exercise. J Orthop Sports Phys Ther, 2008; 38(7): 403-409

Guex K, Millet GP. Conceptual framework for strengthening exercises to prevent hamstring strains. Sports Med, 2013; 43(12): 1207-1215

Heiderscheit BC, Hoerth DM, Chumanov ES, Swanson SC, Thelen BJ, Thelen DG. Identifying the time of occurrence of a hamstring strain injury during treadmill running: a case study. Clin Biomech (Bristol, Avon), 2005; 20(10): 1072-1078

Heiderscheit BC, Sherry MA, Silder A, Chumanov ES, Thelen DG. Hamstring strain injuries: recommendations for diagnosis, rehabilitation, and injury prevention. J Orthop Sports Phys Ther, 2010; 40(2): 67-81

Holcomb WR, Rubley MD, Heather JL, Guadagnoli MA. Effect of hamstring emphasized resistance training 
on hamstring:quadriceps ratio. J. Strength Cond. Res, 2007; 21(1): 41-47.

Hubley-Kozey CL, Deluzio KJ, Landry SC, McNutt JS, Stanish WD. Neuromuscular alterations during walking in persons with moderate knee osteoarthritis. J Electromyogr Kinesiol, 2006; 16(4): 365-378

Iga J, Fruer CS, Deighan M, Croix MD, James DV. 'Nordic' hamstrings exercise - engagement characteristics and training responses. Int J Sports Med, 2012; 33(12): 1000-1004

Lim BO, Lee YS, Kim JG, An KO, Yoo J, Kwon YH. Effects of sports injury prevention training on the biomechanical risk factors of anterior cruciate ligament injury in high school female basketball players. Am J Sports Med, 2009; 37(9): 1728-1734

Lynn SK, Costigan PA. Changes in the medial-lateral hamstring activation ratio with foot rotation during lower limb exercise. J Electromyogr Kinesiol, 2009; 19(3): e197-205

Mjolsnes R, Arnason A, Osthagen T, Raastad T, Bahr R. A 10-week randomized trial comparing eccentric vs. concentric hamstring strength training in well-trained soccer players. Scand J Med Sci Sports, 2004; 14(5): 311-317

Monajati A, Larumbe-Zabala E, Goss-Sampson M, Naclerio F. The Effectiveness of Injury Prevention Programs to Modify Risk Factors for Non-Contact Anterior Cruciate Ligament and Hamstring Injuries in Uninjured Team Sports Athletes: A Systematic Review. PLoS One, 2016; 11(5): e0155272

Naclerio F, Larumbe-Zabala E, Monajati A, Goss-Sampson M. Effects of two different injury prevention resistance exercise protocols on the hamstring torque-angle relationship: a randomized controlled trial. Res Sports Med, 2015; 23(4): 379-393

Opar DA, Williams MD, Shield AJ. Hamstring strain injuries: factors that lead to injury and re-injury. Sports Med, 2012; 42(3): 209-226

Orchard J, Marsden J, Lord S, Garlick D. Preseason hamstring muscle weakness associated with hamstring muscle injury in Australian footballers. Am J. Sports Med., 1997; 25(1): 81-85

Ortiz A, Trudelle-Jackson E, McConnell K, Wylie S. Effectiveness of a 6-week injury prevention program on kinematics and kinetic variables in adolescent female soccer players: a pilot study. P R Health Sci J, 2010; 29(1): 40-48

Pollard CD, Sigward SM, Ota S, Langford K, Powers CM. The influence of in-season injury prevention training on lower-extremity kinematics during landing in female soccer players. Clin J Sport Med, 2006; 16(3): 223-227

Stevenson JH, Beattie CS, Schwartz JB, Busconi BD. Assessing the effectiveness of neuromuscular training programs in reducing the incidence of anterior cruciate ligament injuries in female athletes: a systematic review. Am J Sports Med, 2015; 43(2): 482-490

Thelen DG, Chumanov ES, Best TM, Swason SC, Heiderscheit BC. Stimulating of biceps femoris musculotendon mechanics during the swing phase of sprinting. Med Sci. Sport Exerc, 2005; 37(11): 19311938

Timmins RG, Shield AJ, Williams MD, Lorenzen C, Opar DA. Biceps femoris long head architecture: a reliability and retrospective injury study. Med Sci Sports Exerc, 2015; 47(5): 905-913

Visser JJ, Hoogkamer JE, Bobbert MF, Huijing PA. Length and moment arm of human leg muscles as a function of knee and hip-joint angles. Eur J Appl Physiol Occup Physiol, 1990; 61(5-6): 453-460

Woods C, Hawkins RD, Maltby S, Hulse M, Thomas A, Hodson A. The Football Association Medical Research Programme: an audit of injuries in professional football-analysis of hamstring injuries. Br. J. Sports Med., 2004; 38: 36-41

\section{Corresponding author:}

\section{Dr. Fernando Naclerio}

Centre for Science and Medicine in Sport and Exercise, University of Greenwich Medway Campus Central Avenue, Chatham Maritime, Kent ME4 4TB

Tel: +44(0)20831 8441; Fax: +44(0)208319805

E-mail: f.j.naclerio@gre.ac.uk 